\title{
IMAGEAMENTO DE REFLEXÕES MÚLTIPLAS SIMÉTRICAS EM DADOS SINTÉTICOS UTILIZANDO O MÉTODO DE EMPILHAMENTO SÍSMICO i-CRS
}

\author{
Carlos Eduardo Amanajás Soares, Faculdade de Geofísica, Instituto de Geociências, UFPA
}

Pedro Andrés Chira Oliva, Instituto de Estudos Costeiros (IECOS), UFPA

Copyright 2016, SBGf - Sociedade Brasileira de Geofísica

Este texto foi preparado para a apresentação no VII Simpósio Brasileiro de Geofísica, Ouro Preto, 25 a 27 de outubro de 2016. Seu conteúdo foi revisado pelo Comitê Técnico do VII SimBGf, mas não necessariamente representa a opinião da SBGf ou de seus associados. E proibida a reprodução total ou parcial deste material para propósitos comerciais sem prévia autorização da SBGf.

\section{Resumo}

The simulation of a zero-offset ( $\mathrm{ZO}$ ) seismic section from multi-coverage seismic data is a standard imaging method widely used in seismic processing to reduce the amount of data and increases the signal-to-noise ratio. In the last years, have appeared diverse stacking methods as an extension of the conventional imaging method, the Common-midpoint (CMP) stacking, to simulate ZO sections. Recently an implicit traveltime approximation for monotypic waves in anisotropic media was proposed to simulate ZO sections, called implicit Common-ReflectionSurface (i-CRS). In this work, we tested the performance of this approximation to simulate $\mathrm{ZO}$ seismic sections by considering primary and symmetric multiple reflections.

\section{Introdução}

A simulação de seções zero-offset (ZO) é uma das principais etapas do processamento sísmico. Existem diversos métodos de empilhamento que simulam seções ZO utilizando diversos tipos de aproximações de tempos de trânsito. Dentre os métodos de empilhamento convencionais está o Comom-midpoint (CMP) que organiza os dados ao longo da curva CMP, onde os dados consistem em pares fonte-receptor localizados simetricamente em relação ao CMP. Para empilhar os dados ao longo da curva CMP, este método considera o tempo de trânsito Normal-moveout (NMO), cuja formula é hiperbólica e só depende de um único parâmetro chamado de velocidade de empilhamento ou NMO.

Nas últimas décadas, um variado número de aproximações de tempos de trânsito foi introduzido como uma extensão do método de empilhamento sísmico CMP, onde se destacam o Polystack (e.g. de Bazelaire, 1988), o método Common-Reflection-Surface (CRS, e.g. Müller, 1999; Mann et al., 1999; Garabito, 2001; Jäger et al., 2001), e método Multifoco (MF, e.g. Gelchinsky et al, 1999 a,b; Chira, 2000; Gurevich et al., 2002; Landa et al., 1999). A exatidão destes métodos difere e depende não somente do afastamento, mas também da curvatura do refletor. Outro ponto importante é que nenhum destes métodos considera a anisotropia sísmica.

O método e empilhamento sísmico CRS utiliza uma aproximação hiperbólica multiparamétrica para a superfície de tempos de reflexão (Schleicher et al., 1993; Tygel et al., 1997) também conhecida como aproximação CRS de $2^{\text {a }}$ ordem ou aproximação CRS convencional. O método CRS determina por meio de análise de coerência, três parâmetros: o ângulo de emergência do raio normal (raio central) e os outros dois que são as curvaturas dos eventos sísmicos correspondentes a duas ondas hipotéticas, Normal-Incidence-Point (NIP) e Normal $(N)$ introduzidas por Hubral (1983).

Höcht et al. (1999) derivaram uma expansão de Taylor da aproximação CRS de $2^{\mathrm{a}}$ ordem denominando-a como aproximação CRS de 4⿳亠丷a ordem. Esta nova aproximação é descrita com o mesmo número de parâmetros da aproximação anterior. Oliva et al. (2010) aplicaram satisfatoriamente a aproximação de tempos de trânsito CRS de $4^{\text {a }}$ ordem em dados reais da bacia de Tacutu (Brasil).

Vanelle et al. (2010) introduziram uma aproximação de tempos de trânsito implícita para ondas monotípicas em meios anisotrópicos. Segundo os autores esta aproximação foi derivada a partir da Lei de Snell considerando uma superfície esférica e isto conduziu a uma expressão implícita para a superfície de tempos de trânsito.

Vanelle et al. (2012) aplicaram esta aproximação em meios isotrópicos e anisotrópicos. Os resultados demonstraram uma exatidão superior quando comparados com o método CRS convencional (2a ordem) para uma grande variedade de formas de refletores, desde refletores planos até formas mais complexas com características de gerar eventos de difrações.

Neste trabalho foi aplicado o método de empilhamento iCRS em dados sintéticos para avaliar a performance deste método na simulação de seções $Z O$ considerando eventos de reflexões primárias e múltiplas simétricas.

\section{Método}

Vanelle et al. (2010) e Schwarz (2011) introduziram essa nova aproximação de tempos de trânsito de empilhamento sísmico para ondas monotípicas em meios isotrópicos que leva em consideração uma nova aproximação implícita para avaliar o ponto de reflexão sobre um círculo fazendo uso do princípio de Fermat. Esta aproximação é referida como a aproximação implicit common-reflection-surface (i-CRS) ou superfície de reflexão comum implícita.

Vanelle et al. (2012) consideraram um refletor esférico em um meio homogêneo. $O$ raio do refletor é $R$, com o seu centro no local $\left(x_{c}, 0, H\right)$, como mostrado na Figura 1. $x_{1}$ e $x_{2}$ são coordenadas de uma fonte e de um receptor, respectivamente, ambos na profundidade $z=0$ e $y=0$. O ângulo $\theta$ define o ponto de reflexão em $\vec{r}=(R \operatorname{sen} \theta, 0, R \cos \theta)$. As velocidades do raio dos dois segmentos do raio de ida e volta são $v_{i}\left(\vartheta_{i}\right)$, sendo $\vartheta_{i}$ o ângulo de grupo. 


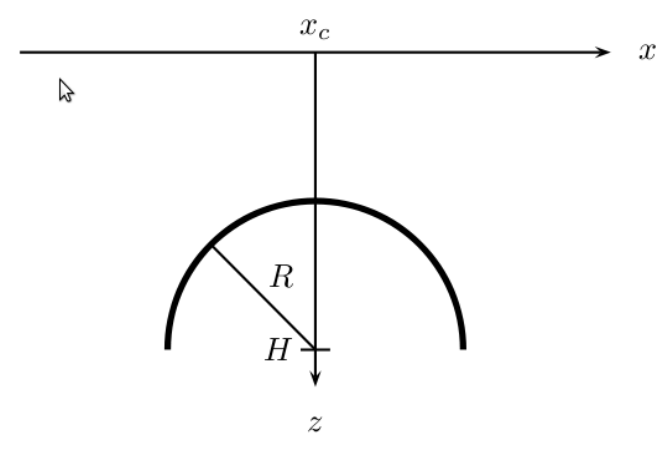

(a)

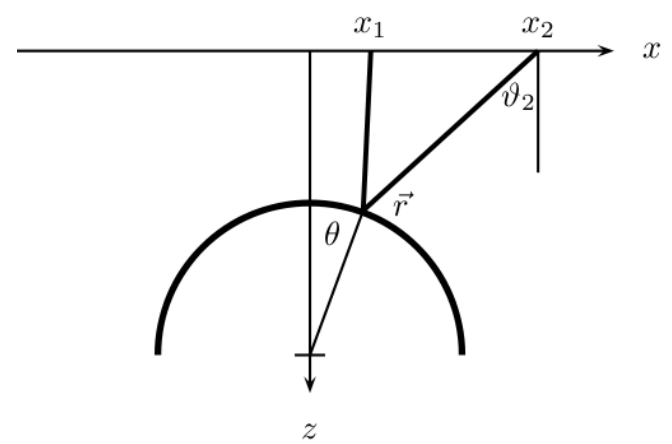

(b)

Figura 1 - a) Geometria do refletor e (b) aquisição para um refletor esférico. O ponto de reflexão é definido pelo ângulo $\theta$. Os ângulos $\vartheta_{i}$ são os ângulos raio/grupo (Vanelle et al. , 2012).

O tempo de percurso $t_{i}$ dos segmentos de raios de ida $\mathrm{e}$ volta são dados por:

$$
t_{i}^{2}=\frac{\left(x_{i}-x_{c}-R \sin \theta\right)^{2}+(H-R \cos \theta)^{2}}{v_{i}^{2}\left(\vartheta_{i}\right)}
$$

ou, em função das coordenadas do ponto médio e meioafastamento $\left(x_{m}, h\right)$ expresso por:

$$
\begin{aligned}
& t_{1}^{2}=\frac{\left(x_{m}-h-x_{c}-R \sin \theta\right)^{2}+(H-R \cos \theta)^{2}}{v_{1}^{2}\left(\vartheta_{1}\right)} \\
& t_{2}^{2}=\frac{\left(x_{m}+h-x_{c}-R \sin \theta\right)^{2}+(H-R \cos \theta)^{2}}{v_{2}^{2}\left(\vartheta_{2}\right)}
\end{aligned}
$$

$$
t_{i}=t_{1}+t_{2}
$$

A soma de $t_{1}$ e $t_{2}$ deve sastifazer a Lei de Snell, ou seja, $\partial\left(t_{1}+t_{2}\right) / \partial \theta=0$.

A aproximação i-CRS também pode ser expressa em função dos três parâmetros do CRS convencionais (Schwarz, 2011).

Para cada amostra $\left(x_{0}, t_{0}\right)$ na seção empilhada, i.e. a seção ZO a ser simulada, temos que determinar os três parâmetros i-CRS que produzem o melhor ajuste para um determinado evento de reflexão a ser simulado. Isto é feito através da análise de coerência (semblance, Taner e Koehler, 1969) ao longo da aproximação de tempos de trânsito i-CRS nos dados de cobertura múltipla.

Após a determinação do trio paramétrico do i-CRS procedemos à aplicação do empilhamento i-CRS.

\section{Resultados}

A fim de testar o desempenho do método de empilhamento i-CRS em relação ao seu potencial para simular eventos de reflexões primárias e, principalmente, múltiplas simétricas, considerou-se um modelo 2-D constituído por duas camadas homogêneas sobre um semi-espaço e separadas por interfaces suavemente curvas (Figura 2). As velocidades para cada camada, desde o topo até a base, são: $2,5 \mathrm{~km} / \mathrm{s}, 3,5 \mathrm{~km} / \mathrm{s}$ e 5,5 $\mathrm{km} / \mathrm{s}$ respectivamente. As dimensões do modelo são: 4 $\mathrm{km}$ de comprimento por $3 \mathrm{~km}$ de profundidade. A aquisição dos dados foi realizada considerando uma linha sísmica horizontal.

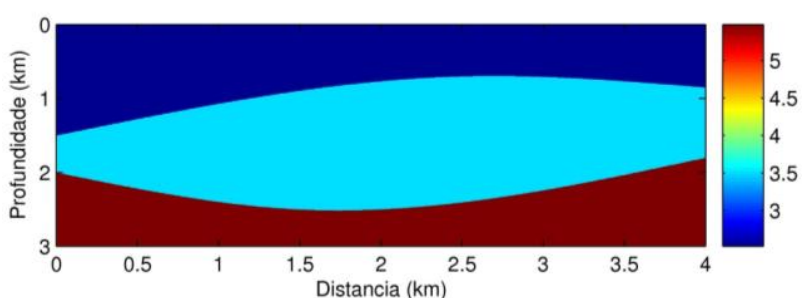

Figura 2 - Modelo 2-D constituído por duas camadas separadas com interfaces curvas e suaves sobre um semi-espaço.

Baseado neste modelo foi gerado um conjunto de dados sintéticos de reflexão de cobertura múltipla utilizando o algoritmo de traçamento de raios, SEIS88 (Cerveny e Psensik, 1988). Os dados não contêm ruído e foram gerados utilizando a configuração common-shot (CS) ou de tiro comum. O mínimo e o máximo afastamento foram de $0,6 \mathrm{~km}$ e 2,9 km respectivamente.

Foi considerada uma fonte tipo Gabor com uma frequência dominante de $50 \mathrm{~Hz}$ e um intervalo de amostragem de tempo de $0,002 \mathrm{~s}$. A primeira fonte foi posicionada em $x_{S}=0,5 \mathrm{~km}$ e primeiro geofone em $x_{G}=$ $1,1 \mathrm{~km}$, sendo distribuídos 48 geofones com espaçamentos de $0,025 \mathrm{~km}$ entre eles. Foram considerados 70 tiros com intervalos de $0,05 \mathrm{~km}$ cada. A seção sísmica zero-offset (ZO) a ser simulada é 
mostrada na Figura 3 e consta de 81 traços com intervalos de $0,025 \mathrm{~km}$.

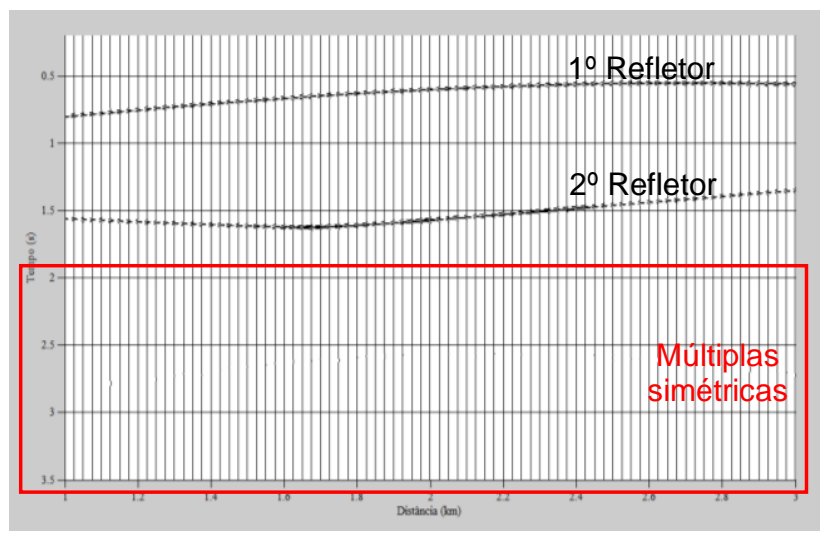

Figura 3 - Seção zero-offset (ZO) original obtida através do traçamento de raios com o software SEIS88. As reflexões múltiplas estão destacadas pelo retângulo vermelho.

O resultado da simulação da seção ZO com o método de empilhamento sísmico i-CRS é apresentado na Figura 4. Podemos observar a presença das reflexões primárias e das reflexões múltiplas (com menor amplitude) geradas na segunda camada. A aproximação i-CRS apresenta um bom desempenho na simulação de eventos de reflexões primárias e múltiplas.

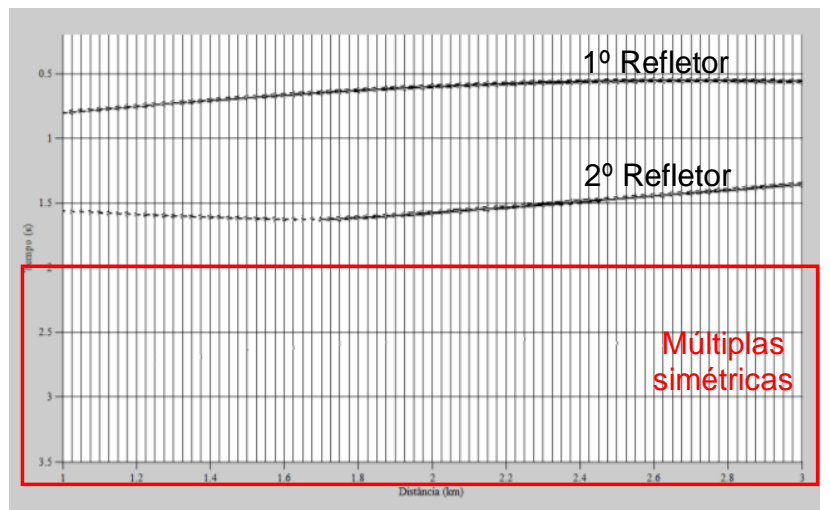

Figura 4 - Seção de afastamento-nulo (ZO) simulada a partir da aplicação do empilhamento sísmico i-CRS. As reflexões múltiplas simétricas estão destacadas pelo retângulo vermelho.

Comparando a seção ZO original (Figura 3) com a seção ZO simulada com o empilhamento sísmico i-CRS (Figura 4) observamos um bom imageamento dos eventos, tanto de reflexões primárias quanto de reflexões múltiplas. Notando-se assim, uma boa definição dos refletores e uma boa aproximação da seção original.

Posteriormente com a finalidade de realçar as reflexões múltiplas simétricas obtidas com o software SEIS88 (Figura 3 ) e as simuladas com o método i-CRS (Figuras 4) foi aplicado um ganho que é visualizado através de um zoom ampliado (Figuras 5 e 6).

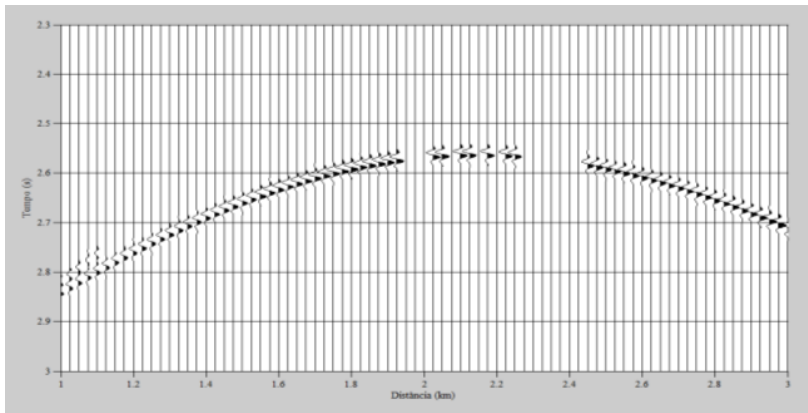

Figura 5 - Trecho destacado em vermelho da Figura 3, mostrando as reflexões múltiplas simétricas obtidas pelo traçamento de raios com o software SEIS88.

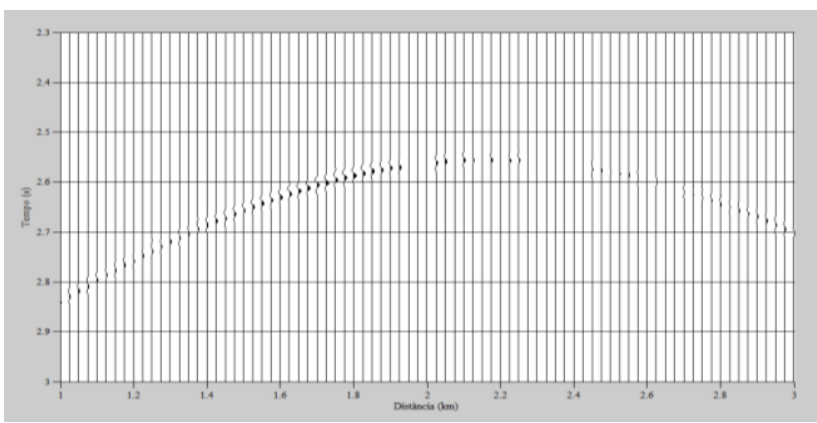

Figura 6 - Trecho destacado em vermelho da figura 6 , destacando as reflexões múltiplas simétricas simuladas pelo empilhamento sísmico i-CRS.

O método de empilhamento i-CRS apresenta uma boa performance na simulação das reflexões múltiplas simétricas (Figura 6) em relação ao sismograma original (Figura 5). A ausência de alguns traços no sismograma se dá devido à ausência de registro pelos receptores como consequência da forma dos refletores.

As Figuras 7, 8 e 9 a seguir, apresentam-se uma comparação entre os traços da seção ZO original e os traços simulados obtidos a partir do empilhamento i-CRS, correspondentes aproximadamente nos flancos esquerdo, centro e direito, esquerdo e na parte central do modelo da Figura 24. As coordenadas dos pontos médios nos locais selecionados são $x_{0}=1,475 \mathrm{~km}$ (Figura 7), $x_{0}$ $=1,975 \mathrm{~km}$ (Figura 8) e $x_{0}=2,6 \mathrm{~km}$, respectivamente (Figura 9), respectivamente. Em geral o método i-CRS apresenta bom desempenho na simulação da forma dos traços ZO, mas não preserva as amplitudes dos mesmos.

O primeiro traço ZO simulado que foi escolhido (Figura 7) corresponde à posição $x=1,475 \mathrm{~km}$ e está localizado aproximadamente no flanco esquerdo do modelo da Figura 2. Na Figura observa-se a boa recuperação da forma do traço da seção ZO original para as reflexões primárias, com uma ligeira diminuição na amplitude do traço correspondente a segunda camada devido à perda de energia por esta se encontrar mais profundo. As reflexões múltiplas simétricas aparecem com amplitudes muito baixas devido à perda de energia com as sucessivas reflexões sofridas antes do seu registro. 
Em relação as reflexões múltiplas simétricas, que perdem energia com a profundidade, as mesmas foram destacadas na Figura 8. Analisando esta Figura observase que os traços simulados recuperaram bem a forma e aumentam a amplitude do traço $\mathrm{ZO}$ original.

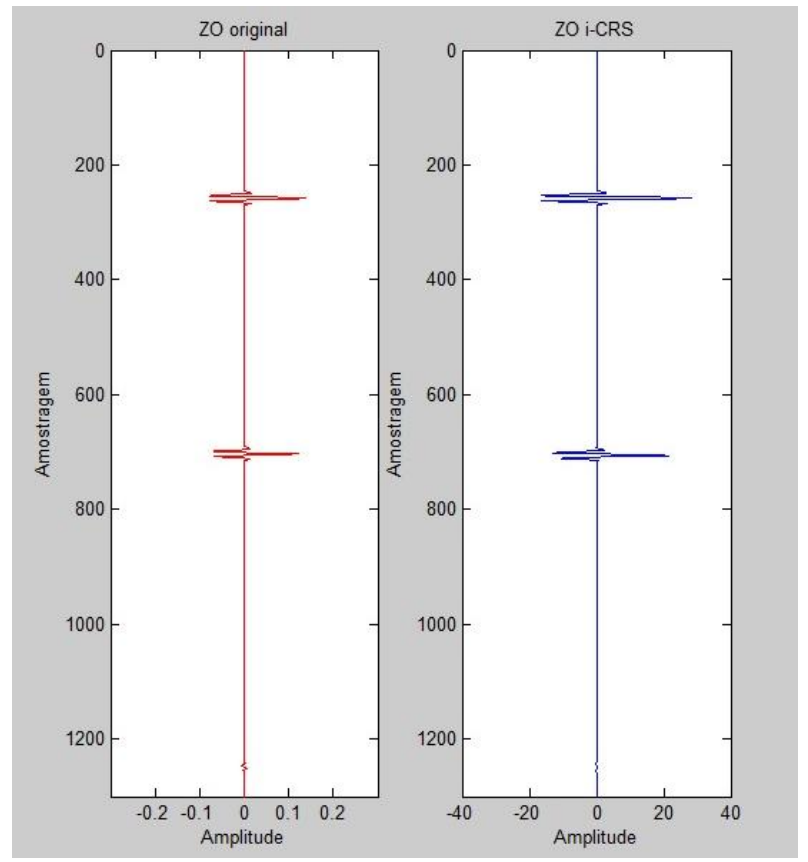

Figura 7 - Comparação do traço sísmico da seção original com o traço correspondente ao empilhamento iCRS. Os traços correspondem a posição $x=1,475 \mathrm{~km}$ na linha sísmica.

Analisando o traço ZO simulado (Figura 9) na parte central do modelo da Figura 2, correspondente a posição $\mathrm{x}_{0}=1,975 \mathrm{~km}$, observamos o mesmo comportamento do pulso anterior. Temos uma boa recuperação da forma dos traços em relação a seção original referentes as reflexões primárias, bem como do traço referente a reflexão múltipla simétrica (Figura 10), pelo método de empilhamento sísmico i-CRS.

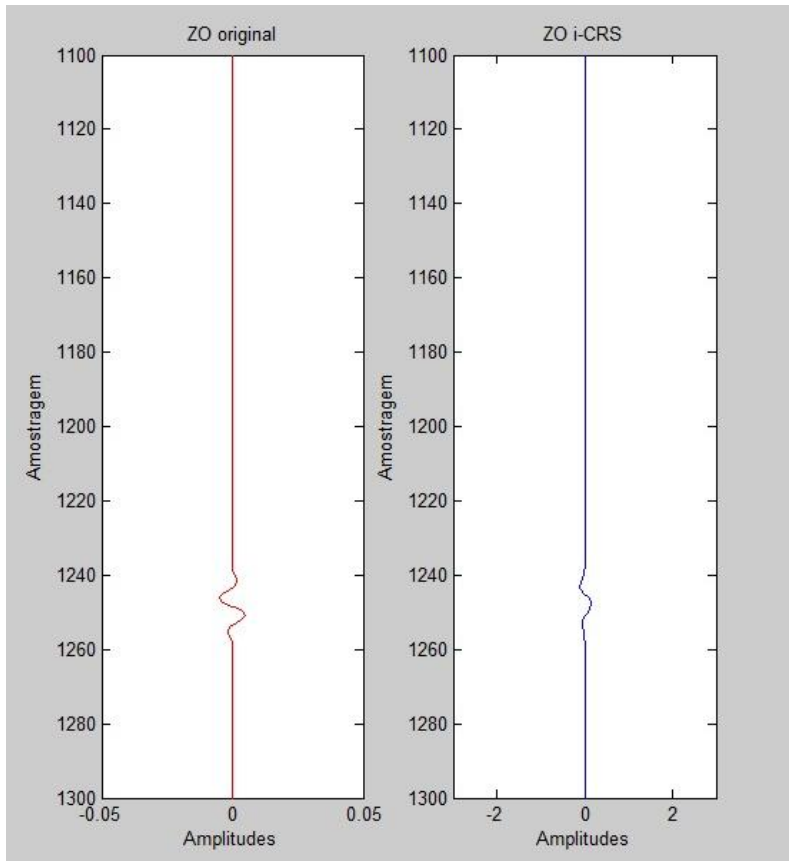

Figura 8 - Comparação do traço sísmico referente apenas às reflexões múltiplas da seção original com 0 traço correspondente ao empilhamento i-CRS. Os traços correspondem a posição $x=1,475 \mathrm{~km}$ na linha sísmica.

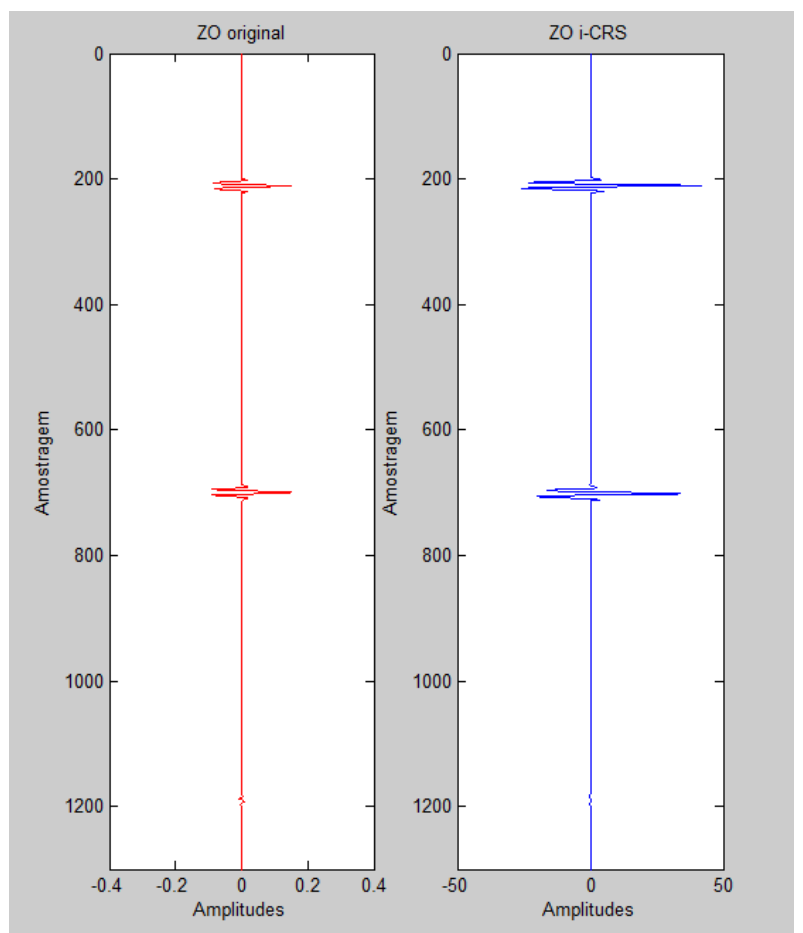

Figura 9 - Comparação do traço sísmico da seção original com o traço correspondente ao empilhamento i-CRS. Os traços correspondem a posição $\mathrm{x}=1,975 \mathrm{~km}$ na linha sísmica. 


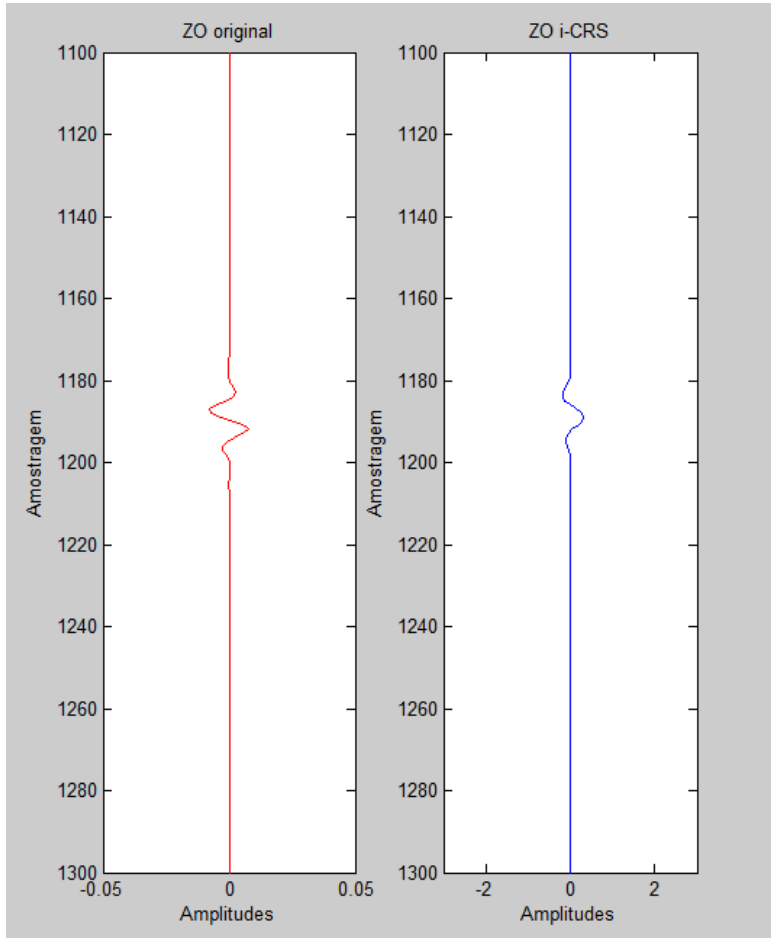

Figura 10 - Comparação do traço sísmico referente apenas às reflexões múltiplas da seção original com o traço correspondente ao empilhamento i-CRS. Os traços correspondem a posição $x=1,975 \mathrm{~km}$ na linha sísmica.

No traço ZO simulado correspondente ao flanco direito do modelo da Figura 2, localizado na coordenada $x_{0}=2,6$ $\mathrm{km}$, observou-se mais uma vez uma boa recuperação dos traços referentes às reflexões primárias obtidos com o método i-CRS (Figura 11).

Quanto as reflexões múltiplas simétricas destacadas na Figura 12, a recuperação dos traços também se mostrou satisfatória com o método i-CRS, já que acompanhou a forma do traço original (com uma menor amplitude).

\section{Conclusões}

A aproximação de tempos de trânsito i-CRS apresenta um bom desempenho na simulação de seções sísmicas ZO levando em consideração as reflexões primárias e múltiplas simétricas. Segundo os resultados obtidos podemos observar um bom posicionamento dos tempos de trânsito e uma boa visualização dos referidos eventos quando comparados com os traços de afastamento-nulo originais.

No que se refere à amplitude e forma dos pulsos das reflexões múltiplas, o método de empilhamento sísmico iCRS se aproxima satisfatoriamente da forma do pulso original levando em consideração diferentes afastamentos em relação à fonte.

O método de empilhamento sísmico i-CRS apresenta-se como uma importante alternativa para simular seções zero-offset (ZO) contendo reflexões múltiplas e primárias para diversos afastamentos.

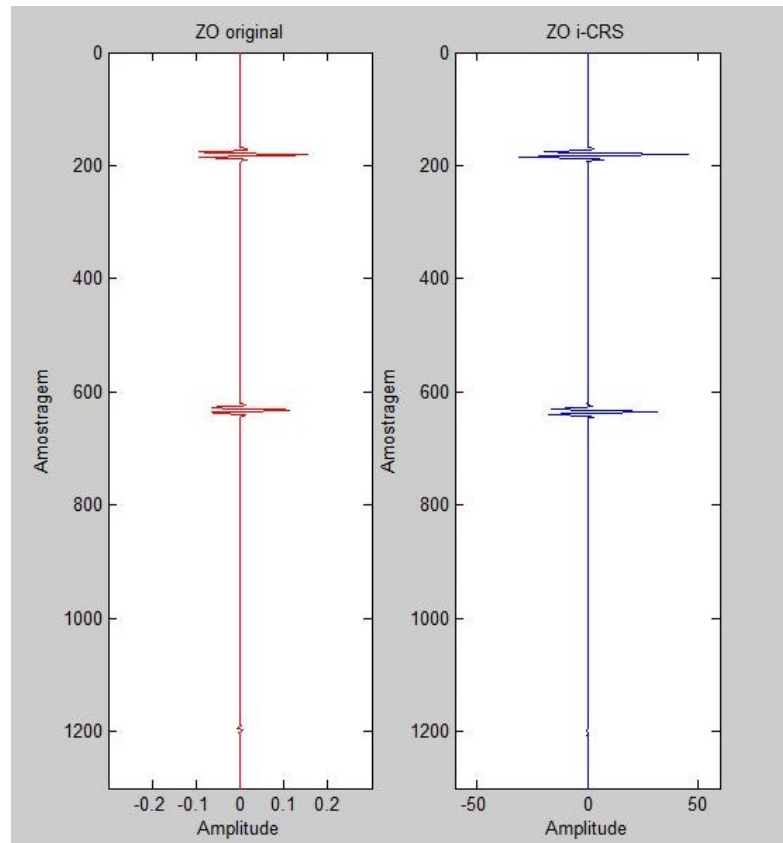

Figura 11 - Comparação do traço sísmico da seção original com o traço correspondente ao empilhamento iCRS. Os traços correspondem a posição $x=2,6 \mathrm{~km}$ na linha sísmica.

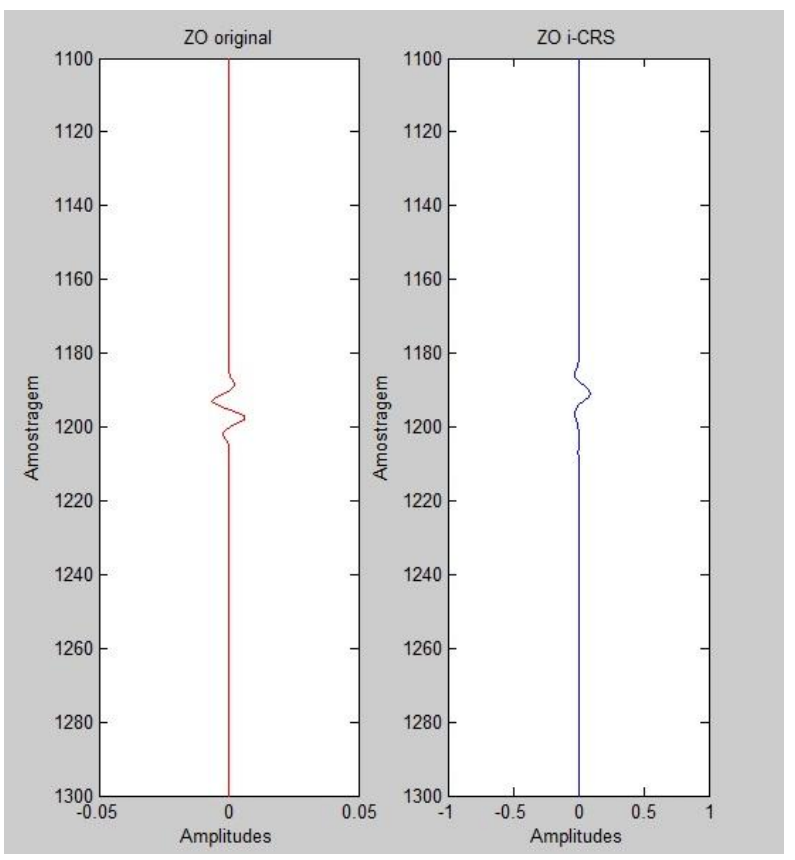

Figura 12 - Comparação do traço sísmico referente apenas às reflexões múltiplas da seção original com o traço correspondente ao empilhamento i-CRS. Os traços correspondem a posição $\mathrm{x}=2,6 \mathrm{~km}$ na linha sísmica.

\section{Agradecimentos}

O primeiro autor gostaria de agradecer ao Programa Institucional de Bolsas de Iniciação Científica (PIBIC/UFPA) pelo suporte financeiro enquanto bolsista. 


\section{Referências}

ČERVENÝ V \& PSENCIK I. 1988. SEIS88, Ray Tracing Program Package.

CHIRA-OLIVA P, TYGEL M, HUBRAL P \& SCHLEICHER J. 2003. A fourth-order CRS moveout for reflection or diffraction events: numerical examples. Journal of Seismic Exploration, 12:197-219.

DE BAZELAIRE E. 1988. Normal moveout revisited inhomogeneous media and curved interfaces. Geophysics, 52, 143-157. FOMEL, S., KAZINNIK, R. 2012. Non-hyperbolic common reflection surface. Geophysical Prospecting, 61: 21-27.

GARABITO G. 2001, Empilhamento Sísmico por Superfície de Reflexão Comum: Um novo Algoritmo Usando Otimização Global e Local, Universidade Federal do Pará. (Tese de Doutorado).

GELCHISNKY B, BERKOVITCH, A \& KEYDAR S. 1999a. Multifocusing homeomorphic imaging: Part 1. Basic concepts and formulae. Journal of Applied Geophyscis, v. 42 , p. 229-242.

GELCHISNKY B, BERKOVITCH, A \& KEYDAR S. 1999b. Multifocusing homeomorphic imaging: Part 2. Multifold data set and multifocusing. Journal of Applied Geophyscis, v. 42, p. 243-260.

GUREVICH B, KEYDAR, S \& LANDA E. 2002. Multifocusing imaging over an irregular topography. Geophysics, v. 67, p. 639-643.

HÖCHT G, DE BAZELAIRE E, MAJER, P \& HUBRAL P. 1999. Seismic and optics: hyperbolae and curvatures. Revita científica "Journal of Seismic Exploration", 12, p. 261-282.

HUBRAL P. 1983. Computing true amplitudes reflections in a laterally inhomogeneous earth. Geophysics, 48, 1051-1062.

HUBRAL P \& KREY T. 1980. Interval Velocities from Seismic Reflection Time Measurements. Soc. Of Exploration Geophysicists. Western Geophysical Company Houston, Texas.

JÄGER R, MANN J, HÖCHT G \& HUBRAL P. 2001. Common Reflection Surface: Image and attributes. Geophysics, 66, 97-109.

LANDA E, GUREVICH B, KEYDAR S \& TRACHTMAN P. 1999. Application of multifocusing method for subsurface imaging: Journal of Applied Geophysics, 42, 283-300.

MANN J, JÄGER R, MÜLLER, T, HÖCHT, G \& HUBRAL P. 1999. Common-reflection-surface stack - a real data example. J. Appl. Geoph., 42(3,4):301-318.
MÜLLER T. 1999. The common reflection surface stack method: Seismic imaging without explicit knowledge of velocity model. Germany, Karlsruhe University (Ph. D thesis).

CHIRA-OLIVA P, GARABITO G \& CRUZ J. 2010. FourthOrder Stacking Method - Examples. In: 72nd EAGE Conference \& Exhibition incorporating SPE EUROPEC 2010, Barcelona. 72nd EAGE Conference \& Exhibition incorporating SPE EUROPEC 2010.

SCHLEICHER J, TYGEL M \& HUBRAL P. 1993. Parabolic and hyperbolic paraxial two points traveltimes in 3D media. Geophysical Prospecting, 41:495-513.

SCHWARZ B. 2011. A new nonhyperbolic multiparameter stacking operator. Diploma thesis. University of Hamburg.

SILVA P, CHIRA P \& CRUZ J. 2012. Simulação de reflexões primárias e múltiplas usando aproximação paraxial de tempos de trânsito CRS de $4^{\mathrm{a}}$ ordem. V Simpósio Brasileiro de Geofísica.

TYGEL M, MÜLLER T, HUBRAL P \& SCHLEICHER J. 1997. Eigenwave based multiparametertraveltime expansions. In: ANNUAL MEETING OF SEG, 67.,Expansed abstracts, 1770-1773.

VANELLE C, BOBSIN M, SCHEMMERT P, KASHTAN B \& GAJEWSKI D. 2012. RSO - A new Multiparameter Stacking Operator for An/lsotropic media. 74th EAGE Conference \& Exhibition incorportating SPE EUROPEC 2012. Copenhagen, Denmark.

VANELLE C, KASHTAN B, DELL \& GAJEWSKI D. 2010. A new stacking operator for curved subsurface structures (SPNA 3.2). 80th Ann. Int. Mtg. Soc. Expl. Geoph., Expanded Abstracts. 